\title{
ATMOSPHERIC AIR PROTECTION TECHNOLOGIES IN POWDER METALLURGY
}

\author{
Yurij Derkach ${ }^{1}$ \\ Yevhenij Poznij ${ }^{2}$
}

DOI: https://doi.org/10.30525/978-9934-588-52-5-23

The main pollutants in metallurgical production, the concentration of which exceeds the established maximum permissible concentration values are: nitrogen oxide (class 3), carbon monoxide (class 4), abrasive metal dust, $\mathrm{SiO}_{2}$ dust (class 3) and inorganic dust with $\mathrm{SiO}_{2}$ content (class 3). Therefore, removal of these emissions should be provided in 3 stages: 1 - dust removal, 2 - CO removal, 3 - purification from $\mathrm{NO}_{\mathrm{x}}$.

Dust emissions from traction machines contain a significant amount of solid particles and corrosive gases (such as silicon dioxide $\mathrm{SiO}_{2}$, metal shavings and other aggressive components) and are characterized by corrosion and increased abrasiveness. The following applications of the captured dust containing $\mathrm{SiO}_{2}$ are available.

Burial. Disposal of solid waste by burying it is one of the most common ways to get rid of garbage today. However, the disadvantages of landfilling are that the amount of municipal solid waste is constantly growing both in absolute terms and per capita, and the composition of municipal solid waste is sharply complicated, including an increasing number of environmentally hazardous components that pollute water, soil and air, which increases the area of contaminated land in the country.

Disposal for production purposes. This is one of the most common and rational methods of ensuring the waste-free production with a simultaneous increase in its efficiency and the solution of environmental problems. The technology of returning dust that is captured in the main production is usually determined by the methods used for gas cleaning (dry, wet, one- and two-stage, combined) and the feasibility of introducing these products into a specific apparatus of the technological scheme in a particular state.

The dust collected has the physical properties of sand and can be used in general construction work, in the production of concrete and also for the manufacture of abrasive materials, for example for the manufacture of grinding wheels for grinding machines.

The processes of catalytic oxidation of hydrocarbons are carried out in most cases in a continuous scheme. The nature of the initial oxidation

\footnotetext{
${ }^{1}$ National University of Civil Defence of Ukraine, Ukraine

${ }^{2}$ National University of Civil Defence of Ukraine, Ukraine
} 
reactions strongly depends on the temperature level. Thus, during the oxidation of the limiting hydrocarbon at room or moderately elevated temperatures, hydroperoxide can be formed, and at higher temperatures, hydrogen peroxide occurs instead.

As a result of this process, hot gaseous combustion products are formed, which are to be sent to the heat exchanger. The heat exchanger, in turn, emits heat energy, which can be used for air heating or other needs, which can be consumed by plant workers.

Gas stream purified from carbon monoxide enters the catalytic converter of nitrogen oxides. The catalytic converter is installed in the exhaust system to reduce the content of harmful substances in the exhaust gases.

The neutralizer contains one reducing (rhodium) and two oxidizing (platinum and palladium) catalysts. Oxidation catalysts contribute to the oxidation of unburned (if any) in the previous block of hydrocarbons in water vapor, and carbon monoxide in carbon dioxide. The reducing catalyst reduces harmful oxides of nitrogen $\mathrm{NO}_{\mathrm{x}}$ to harmless nitrogen. Since these neutralizers reduce the content of three harmful substances in the exhaust gases, they are called three-component.

After the neutralization process, a solution of nitric acid $\mathrm{HNO}_{3}$ is formed, which is transferred to the pickling shop for further use according to technological processes.

After passing the devices of the entire technological scheme, the gas flow enters the receiver of gas emissions, i.e. into the atmosphere.

\section{ENVIRONMENTAL PROTECTION TECHNOLOGIES IN THE PRODUCTION OF SODIUM HYPOCHLORITE}

\section{Svitlana Kovalenko ${ }^{1}$ Maryna Gorbulja ${ }^{2}$}

DOI: https://doi.org/10.30525/978-9934-588-52-5-24

Today, regulatory and environmental acts have tightened requirements for the quality of drinking water. Sodium hypochlorite, in comparison with other chlorine-containing agents, is the least scarce and fairly cheap detoxifier. Due to its high antibacterial activity and a wide spectrum of action on various microorganisms, sodium hypochlorite is listed first in the list of chlorine

\footnotetext{
${ }^{1}$ National University of Civil Defence of Ukraine, Ukraine

${ }^{2}$ National University of Civil Defence of Ukraine, Ukraine
} 